\title{
METODOLOGÍAS PARTICIPATIVAS CON PERSONAS CON DISCAPACIDAD INTELECTUAL - UNA REVISIÓN TEÓRICA DE LITERATURA
}

\author{
María Gómez-Carrillo de Castro \\ Instituto Superior de Ciências Sociais e Políticas, Universidade de Lisboa, Portugal \\ mariagomez-carrillo@edu.ulisboa.pt
}

\begin{abstract}
Resumen. Esta revisión teórica de literatura recoge las distintas metodologías identificadas como participativas con personas con discapacidad. La participación de personas con discapacidad ha sido reclamada por académicos, personas con discapacidad e incluso por textos legales como la Convención sobre los Derechos de las Personas con Discapacidad. El objetivo de esta revisión teórica de literatura es recopilar los distintos enfoques metodológicos e identificar las barreras, críticas y nuevas experiencias de investigación con personas con discapacidad intelectual. Se identifican los principios comunes de las metodologías participativas, las estrategias para superar dificultades y se encuentran ejemplos concretos de investigaciones con resultados positivos en los que se ha involucrado a personas con discapacidad intelectual de manera significativa.
\end{abstract}

Palabras clave: Participación; Discapacidad Intelectual; Metodología Participativa; Emancipadora.

\section{PARTICIPATORY METHODOLOGIES WITH PERSONS WITH INTELLECTUAL DISABILITIES -} A THEORETICAL LITERATURE REVIEW

\begin{abstract}
This theoretical literature review includes the different methodologies identified as participatory with persons with disabilities. The participation of persons with disabilities has been demanded by academics, persons with disabilities and even by legal texts such as the Convention on the Rights of Persons with Disabilities. The objective of this theoretical literature review is to compile the different methodological approaches and to identify barriers, criticisms and new experiences of research with persons with intellectual disabilities. It identifies common principles of participatory methodologies, strategies to overcome difficulties and finds concrete examples of research with positive results in which persons with intellectual disabilities have been involved in a significant way.
\end{abstract}

Keywords: Participation; Intellectual Disabilities; Participative Methodology; Emancipatory.

\section{INTRODUCCIÓN}

La Convención sobre los Derechos de las Personas con Discapacidad introduce un modelo de derechos humanos y social de la discapacidad y pone especial énfasis en la participación de personas con discapacidad en todas las esferas de la vida, incluida la investigación social. Sin embargo, las personas con discapacidad intelectual han sido tradicionalmente excluidas de la participación en la investigación. Esta revisión teórica de literatura resume las diferentes metodologías existentes y cómo casan con distintos modelos de discapacidad propuestos. En el peor de los casos, se trata de una participación simbólica, en el mejor, de una experiencia enriquecedora para todas las personas implicadas y resultados que contribuyen al avance de la agenda colectiva de las personas con discapacidad. Este trabajo 
forma parte de un proyecto de doctorado (DARE Research ${ }^{1}$ ), financiado por la Unión Europea a través del programa de investigación e innovación Horizon 2020. Se explorará cómo hacer investigación participativa basada en derechos humanos con personas con discapacidad intelectual a partir de dos experiencias participativas en España e Irlanda.

Esta revisión teórica de literatura se centra en las metodologías participativas con personas con discapacidad, con especial énfasis en las personas con discapacidad intelectual. Se describen las distintas modalidades de investigación participativa seguido de una descripción de las barreras, dificultades y críticas reconocidas por la comunidad académica. Las metodologías participativas pueden distinguirse por área de conocimiento, por intensidad de la participación, por el resultado deseado o por el grupo incluido. Las metodologías participativas pretenden atajar respuestas políticas o diseños de programas que no tienen en cuenta las preferencias de las personas o que se fundamentan en investigaciones que no han conseguido reflejar de manera precisa la información sobre el contexto y las personas en el que van a actuar (Cornwall y Jenkes, 1995; Wolff, 2011). No obstante, las personas con discapacidad intelectual rara vez son consultadas (Mietola et al., 2017; Pallisera Díaz et al., 2015) u oídas en la investigación. Entre los retos identificados, se hallan barreras, actitudinales y sociales, además de falta de experiencias con personas con discapacidad intelectual que hayan producido el suficiente material para facilitar la adopción de estrategias y metodologías tendentes a promover la participación de este grupo.

\section{METODOLOGÍA}

Esta revisión teórica ha sido realizada por una única investigadora con el fin de explorar qué se entiende por investigación participativa y cómo se ha formulado en proyectos anteriores con adultos con discapacidad intelectual. No pretende ser una revisión de literatura exhaustiva ni una valoración de los estudios participativos previos, si no más bien atiende a la discusión metodológica que surge de la teoría y práctica.

Esta revisión sistematiza los distintos estudios y metodologías en función de distintos criterios con el fin de establecer una base para el proyecto de doctorado. Se identifican puntos en común y discrepancias y las implicaciones epistemológicas de cada propuesta. Se trata de un proyecto interdisciplinar (ciencias jurídicas y política social), por lo que el foco está en qué metodologías pueden contribuir al conocimiento en estas dos ramas científicas.

\footnotetext{
${ }^{1}$ Dareresearch.eu
} 
Las bases de datos consultadas a través de b.on incluyen: Academic Search Complete, JSTOR, ERIC, Sociological Abstracts, Psyclnfo, Elsevier, Springer, Taylor \& Francis, Wiley. Además, se realizó una búsqueda en google scholar para identificar artículos que no se hallaran en las bases de datos específicadas. La búsqueda se realizó en inglés con los términos de búsqueda "Participatory research" "Inclusive research" "Persons with intellectual disabilities" "developmental" o "CRPD". Se incluyeron todos aquellos artículos específicos sobre metodologías participativas con personas con discapacidad intelectual, aquellos que se enmarcaran como análisis teóricos de metodologías participativas con personas con discapacidad, con un fuerte componente metodológico o que incluyeran una discusión entorno a estas metodologías.

Se excluyó la investigación realizada con menores, la investigación participativa en el ámbito de las ciencias de la salud y la educación dado que no siempre está garantizado que partan de un concepto social de la discapacidad. Además, se buscó específicamente aquellos artículos referidos discutieran la metodología participativa con personas con discapacidad. Durante la selección de artículos, se atendió a las siguientes preguntas:

1. ¿Discute la metodología participativa con personas con discapacidad (intelectual) en alguna de sus modalidades?

2. ¿Cumple con la definición de participativa (más allá de ser entrevistados) y con un intento de compartir el proceso de toma de decisión sobre la investigación?

3. ¿Parte de un modelo social de discapacidad?

La búsqueda se realizó entre noviembre 2019 - febrero 2020.

Los artículos fueron clasificados a través de una tabla excel en la que se desagregaba la información para facilitar su análisis, destacándose las definiciones y criterios propuestos por los autores.

\subsection{Clasificación de las metodologías participativas}

\subsubsection{Por grupo incluido}

En la investigación participativa basada en la comunidad se parte de la comunidad como unidad, como indica el primer principio orientador (Israel et al., 1998; Minkler y Wallerstein, 2008). Las personas con discapacidad y, en especial las personas con discapacidad intelectual, han sido invisibilizadas y excluidas de los espacios comunitarios (Mietola et al., 
2017). Se reclamó, por tanto, una investigación participativa liderada por las personas con discapacidad en lugar de realizar investigación sobre las personas (Oliver, 1992). Mike Oliver, autor del modelo social de la discapacidad, reclamó que los estudios realizados sobre discapacidad fueran liderados por personas con discapacidad como auténticos expertos en discapacidad. La propia disciplina de disability studies nace de una necesidad de formas alternativas de conocimiento en relación con la discapacidad y las personas con discapacidad (Mietola et al., 2017). Esta disciplina parte de un fuerte modelo de derecho de la discapacidad y un compromiso político para erradicar la discriminación e desigualdad social.

Las metodologías participativas buscan dar voz a los que rara vez son oídos e involucrar a las personas para invertir los procesos de aprendizaje y obtención de datos sobre las personas con discapacidad. Como se explicará más adelante, dentro de las metodologías participativas con personas con discapacidad se ha diferenciado entre metodologías participativas, emancipadoras e inclusivas. La última hace referencia a la inclusión de personas con discapacidad intelectual, que raramente habían sido consultados o incluidos en la investigación tradicional ni la investigación participativa o emancipadora (Milner y Kelly, 2009; Mietola et al., 2017).

Ha recibido una metodología propia por esa falta de inclusión en la investigación emancipadora defendida por otros investigadores con discapacidad y porque, el modelo social, entendido en su sentido estricto y por los autores anglosajones, negaba la propia experiencia de la deficiencia (Morris, 1991) y no refleja bien las experiencias de las personas con discapacidad intelectual. Como respuesta a esta interpretación del modelo social, existe también el modelo nórdico (Gustavsson et al., 2005) con una idea más relacional de autonomía y el modelo de la discapacidad de derechos humanos (Degener, 2015), que también ha desarrollado su propuesta de metodología participativa, como se verá más abajo.

\subsubsection{Por intensidad}

Las formas de participación varian en función de en qué fase de la investigación se incluye a las personas con discapacidad y con qué intensidad. Varios autores han propuesto distintos grados o clasificaciones (Bigby et al., 2014; Zarb, 1992). Biggs (1989) propone cuatro modalidades: contractual, en la que las personas son contratadas por el equipo investigador para participar en su proyecto; consultativa, en la que las personas opinan antes de que el 
investigador actúe; colaborativa, en la que el investigador y las personas participantes trabajan conjuntamente en un proyecto diseñado, iniciado y gestionado por el investigador; y colegiada, en la que el investigador y las personas participantes trabajan como iguales con diferentes habilidades que ofrecer, en un proceso de aprendizaje mutuo en el que los participantes tienen el control sobre el proceso.

La participación frecuentemente se articula mediante los grupos consultivos. Las personas son consultadas para opinar sobre distintos aspectos de la propuesta y sobre la propia investigación, los investigadores pueden disponer que la temática y pregunta de investigación sea determinada por las personas con discapacidad y sus organizaciones.

Existen investigaciones en las que se incluye a las personas con discapacidad o sus organizaciones como parte de un consejo de investigación, a la que se consulta en diferentes tramos de la investigación. Depende del poder de decisión e impacto que tenga este consejo, la investigación puede considerarse más o menos al servicio de las personas con discapacidad.

Bigby et al. (2014) distinguieron entre la incorporación como grupo de asesoramiento (advising), dirección y control (directing and leading) y colaboración (collaborative group). Otra modalidad es si las organizaciones o grupos de personas con discapacidad establecen las prioridades de investigación o son involucradas en la difusión de los resultados (Pallisera Díaz et al., 2015).

El siguiente grado de participación consiste en investigaciones realizadas por las personas con discapacidad dentro de sus organizaciones (Ward y Simons, 1998). El tercer grado, identificado como tal por Pallisera Díaz et al. (2015), es la co-investigación con personas con discapacidad. En este tipo de investigaciones, las personas con y sin discapacidad trabajan conjuntamente en la elaboración de la investigación, y se respeta al máximo la voz de las personas con discapacidad, que retienen el control sobre sus experiencias, cómo son representadas e interpretadas.

\subsubsection{Por resultado}

La investigación de acción participativa, originalmente ideada para el trabajo de campo en proyectos de cooperación al desarrollo o investigación indígena y la investigación emancipadora. En el caso de las personas con discapacidad, en los años 80, hubo personas con discapacidad que reclamaron contra las investigaciones en las que participaban, pues 
sentían que únicamente beneficiaban al investigador, o que los investigadores estaban de parte de las instituciones que oprimen a las persona con discapacidad. Un ejemplo de esto es la investigación sobre la vida en instituciones en la que los residentes tenían puestas la esperanza de que la investigación arrojara luz sobre la situación que vivían y les apoyara en sus reivindicaciones. Sin embargo, resultaron decepcionados y vieron en los investigadores posturas tibias y alineadas con quienes consideraban sus opresores (Mercer, 2002). No obstante, son varios los autores que reconocen que la emancipación no se puede prever a través de la investigación, puesto que depende del propio sujeto. La investigación puede, en todo caso, contribuir y facilitar a dicho proceso iniciado por el individuo (Stone y Priestley, 1996; Oliver, 1992).

\section{LA INVESTIGACIÓN PARTICIPATIVA}

La diferencia clave entre la investigación participativa y la investigación tradicional radica en la localización del poder durante el proceso de investigación (Cornwall y Jenkes, 1995). Se destaca, además, la proximidad al 'mundo real', en lugar de permanecer en espacios teóricos y académicos (Hohmann y Shear, 2002). En el ámbito de la intervención social, de la salud y de las acciones al desarrollo, se conoce también la investigación de acción participativa, cuyo objetivo es involucrar a determinados colectivos sobre los que actuarán proyectos y políticas participen en su diseño y en las accione de implementación. La investigación participativa basada en la comunidad (CBPR por sus siglas en inglés) se fundamenta también en movimientos de liberación y empoderamiento (Freire, 1973; Fals Borda, 1998) y ha influido la investigación participativa en los estudios de discapacidad.

Los modelos de discapacidad existentes (médico, social anglosajón y social nórdico relacional o la teoría de la normalización) también tienen un impacto sobre la modalidad de metodología participativa. El modelo social tal y como es entendido por autores anglosajones, que con frecuencia no aplica a personas con discapacidad intelectual y corre el riesgo de marginar a este grupo en la investigación (Walmsley, 2001).

Como alternativa, existe la teoría de la normalización, con un fuerte enfoque en derechos humanos en su concepción nórdica, o con influencia de la teoría de la desviación norteamericana. Esta teoría defiende la adaptación de todos los recursos para que las vidas de las personas con discapacidad se asemejen al máximo a una vida ordinaria y el uso de recursos culturales para facilitar, establecer y mantener roles sociales valiosos para las personas con discapacidad (Walmsley, 2001). 
La investigación emancipadora da respuesta al mismo reclamo de pasar a una investigación participativa, a dar voz y control a las personas con discapacidad, pero se distingue en su objetivo: emancipar o empoderar a las personas con discapacidad como colectivo e individualmente a través de la investigación. Se busca dejar de ser objeto o incluso víctima, según algunos autores, de estudios, para pasar a controlar el objeto, la metodología, el análisis y los resultados de las investigaciones, a fin de ajustarlos a las necesidades del colectivo. Zarb (1992) señala que una de las diferencias esenciales entre las metodologías participativas y la emancipadora radica en los principios de empoderamiento y reciprocidad. Reconoce que, el empoderamiento de las personas con discapacidad no va a llegar únicamente a través de la investigación. En línea con la argumentación de Oliver (1992), el empoderamiento no es algo que se pueda dar, si no que depende de la propia persona. Cuando una persona decide embarcarse en un proceso, debe facilitarse por parte de los investigadores. Eso implica, según Oliver, que las relaciones sociales de la investigación deben modificarse, para poner el conocimiento y las habilidades de los investigadores a disposición de las personas con discapacidad.

Para evaluar las investigaciones, Zarb (1992) propone una serie de preguntas (traducción de la autora):

(1) ¿Quién controla sobre qué versará la investigación y cómo se realizará?

(2) ¿Cómo de lejos hemos llegado involucrando a personas con discapacidad en el proceso de investigación?

(3) ¿Qué oportunidades existen para que las personas con discapacidad puedan criticar la investigación e influir sobre direcciones futuras?

(4) ¿Qué ocurrirá con los productos de esta investigación?

El autor coincide con otros autores como Stone y Priestley (1996) en la necesidad de ceder el control a las personas con discapacidad y la necesidad de transparencia y fácil acceso a las investigaciones, además de la necesaria sumisión a posibles críticas. Estos autores también proponen una serie de principios orientadores (traducción de la autora):

- ' 'La adopción del modelo social de la discapacidad como base epistemológica de la investigación;

- La renuncia a las reivindicaciones de objetividad a través de un compromiso político abierto con la lucha de las personas con discapacidad por su emancipación; 
- la voluntad de realizar investigaciones sólo cuando sean de beneficio práctico para el empoderamiento de las personas con discapacidad y/o la eliminación de las barreras que las incapacitan;

- la evolución del control sobre la producción de la investigación para asegurar la plena responsabilidad ante las personas con discapacidad y sus organizaciones;

- dar voz a lo personal como político, y, al a vez, tratar de crear una voz común en lo político de las experiencias individuales;

- la voluntad de adoptar una pluralidad de métodos para la reunión y el análisis de datos en respuesta a las necesidades cambiantes de las personas con discapacidad".

La propuesta de Stone y Priestley (1996) lleva el compromiso político un poco más lejos y exige, por un lado, la adopción del concepto social de discapacidad y la renuncia a la objetividad tan reclamada por el positivismo, que en materia de discapacidad con frecuencia utiliza el modelo médico de discapacidad.

\section{LA INVESTIGACIÓN INCLUSIVA}

La investigación inclusiva se refiere a la inclusión de personas con discapacidad intelectual ${ }^{2}$ como co-investigadores. No es un término cuyo uso esté muy extendido (Seale et al, 2015) y se suele asociar con un modelo de normalización de la discapacidad (Pallisera Díaz et al., 2015). Fue acuñado por Jan Walmsley (2001). Se optó por otro término diferente para señalar que se trata de personas con discapacidad intelectual las que participan. Se fundamenta en la teoría de la normalización y en el modelo social, aceptando las críticas al mismo, y en algunos casos, entendiendo la participación inclusiva como un modo de contribuir a la discusión y construcción de estos conceptos (Boxall, 2007). Walmsley (2001) define la participación inclusiva como más amplia que los conceptos de investigación participativa y emancipadora, en el que las personas con discapacidad intelectual participan más allá de ser entrevistados, y pudiéndose enmarcar en el enfoque participativo o emancipador.

\footnotetext{
2 En el Reino Unido, se diferencia entre personas con dificultades de aprendizaje y discapacidad intelectual. En la comunidad internacional, es más común referirse a este colectivo con el término "discapacidad intelectual". En España, es el término común más empleado, por lo que en este artículo se emplea esta expresión.
} 
Walmsley sostiene que, para ser considerado investigación inclusiva (sea participativa o emancipadora), se deben cumplir los siguientes requisitos (traducción de la autora):

- El problema de investigación debe ser de las personas con discapacidad intelectual. La iniciativa no tiene que salir necesariamente de las personas con discapacidad intelectual, pero si debe responder a sus intereses y necesidades.

- El proyecto debe contribuir a promover los intereses de las personas con discapacidad intelectual, con investigadores sin discapacidad trabajando para y con personas con discapacidad intelectual.

- Debe ser colaborativo e implicar a las personas con discapacidad intelectual a lo largo de todo el proceso.

- La pregunta de investigación, el proceso y los informes resultantes deben ser accesibles para personas con discapacidad intelectual.

No es muy frecuente que existan plataformas $u$ organizaciones únicamente de personas con discapacidad, salvo en el contexto anglosajón (People First organisations como por ejemplo Change People UK). Los grupos de autogestores (selfadvocacy) suelen ser un paso previo y son espacios en los que las personas con discapacidad intelectual hablan con voz propia y defienden sus intereses.

Las formas más comunes de involucrar a personas con discapacidad son como grupos asesores de personas con discapacidad, que son consultados con relativa frecuencia (Durell, 2016) durante la total duración de la investigación. En estos casos, es importante que estos grupos asesores retengan control sobre la investigación, en lugar de convertirse en consultas simbólicas sin impacto alguno. En España, Pallisera Díaz et al. (2015) identificaron varios estudios realizados con metodología participativa y destacaron su escasa aplicación, a pesar de la demanda existente. Este mismo equipo realizó un estudio sobre el proceso de transición a la vida adulta, en el que se incluyó a personas con discapacidad como consejo asesor.

Estas investigadoras destacan, además, que a pesar de ser una de las barreras más descritas, existe muy poca literatura sobre las estrategias para ofrecer apoyos a los investigadores con discapacidad intelectual. En este proyecto se realizaron grupos focales, siguiendo el ejemplo de otras investigaciones con personas con discapacidad intelectual. Las autoras destacan que, para constituir grupos focales con personas con discapacidad 
intelectual, las personas deben tener capacidad reflexiva y capacidad para mantener conversaciones, los temas de discusión deben ser de interés para las personas, el espacio debe ser agradable y conocido, el encuentro debe estar bien planificado con suficiente antelación, establecer normas de funcionamiento de grupo conjuntamente al comenzar la reunión y destinar suficiente tiempo y material para la elaboración de los materiales de apoyo y la preparación.

Existen también investigaciones de historias de vida (Johnson y Traustadóttir, 2005) o experiencias junto con personas con discapacidad intelectual, en el que las personas con discapacidad intelectual fueron involucradas como co-autoras, respetándose su interpretación de la misma a través de parejas (una persona con discapacidad y una persona sin discapacidad que ayuda a enmarcar y responder a la experiencia de la persona con discapacidad en el marco de la Convención sobre los Derechos de las Personas con Discapacidad). ${ }^{3}$ En este proyecto, el requisito inicial era aceptar el marco teórico de la Convención y tener una historia propia de una vulneración de uno de los derechos recogidos en la Convención y querer contarla.

La relación entre el investigador sin discapacidad y el investigador con discapacidad es controvertida. Algunos autores asumen una desigualdad inherente en esta relación, mientras que otros aceptan que puede ser modulada en función de cómo se establezca la relación y se desarrolle la investigación. El apoyo de otras personas sin discapacidad es considerado un riesgo por si toman las riendas de la investigación. Sin embargo, ha habido experiencias variadas (Durell, 2016). Se diferencia entre un enfoque de "la persona primero", en el que las personas de apoyo están en un segundo plano y sólo intervienen si fuera necesario, o se las entiende como parte del grupo investigador (Durell, 2016). Las publicaciones entorno a estrategias para crear un grupo de trabajo también son escasas (Pallisera Díaz et al., 2015).

El grupo irlandés de investigación inclusiva (Irish Inclusive Research Network) define la investigación inclusiva ${ }^{4}$ como (traducción de la autora):

- “Un espacio en el que las personas con discapacidad intelectual reciben el apoyo para llevar a cabo su propia investigación.

- Se comparte el poder: las personas con discapacidad participan en todas las tomas de decisiones en todas las fases del proyecto.

\footnotetext{
${ }^{3}$ Ercvoices.com, Proyecto liderado por Eilionóir Flynn, Centre for Disability Law and Policy, NUI Galway.

${ }^{4}$ http://www.fedvol.ie//nclusive Research Network IRN/Default.241.html
} 
- Se construye una comunidad de investigación (...)."

Se destaca de esta definición la idea de construir una comunidad de investigadores. Coincide con otros autores en el especial valor a las relaciones personales con los investigadores (Seale et al., 2015).

\section{METODOLOGÍAS PARTICIPATIVAS BASADAS EN DERECHOS HUMANOS}

Las metodologías participativas basadas en derechos humanos proponen aunar todos los principios de las demás metodologías y utilizar el marco teórico de los derechos humanos, con el reconocimiento inherente de la dignidad de la persona.

Se distinguen de las metodologías anteriores en cuanto asumen la Convención sobre los Derechos de las Personas con Discapacidad como marco teórico. Coinciden en la necesidad de dejar a un lado la exigencia de objetividad y ven un compromiso personal, político y profesional con las personas con discapacidad. La Red de Investigación de Discapacidad y Derechos Humanos (Disability Human Rights Research Network, DHRRN) fue creada por la Universidad de Melbourne ${ }^{5}$, Australia, y establece una plataforma para fomentar la investigación académica entre investigadores de derechos humanos y discapacidad y entidades defensoras de derechos humanos, personas con discapacidad y sus organizaciones. Esta red (DHRRN) articula además una serie de protocolos y guías para informar la investigación, como por ejemplo una guía sobre cómo garantizar la accesibilidad (virtual y en papel) de los resultados de las investigaciones ${ }^{6}$. Los principios informadores de esta propuesta metodológica incluyen:

- Una investigación iniciada y liderada por la comunidad de la discapacidad

- Una investigación que responda a una preocupación jurídica de la comunidad de la discapacidad

- Una investigación cuyos resultados (outputs) respondan a esa preocupación jurídica y sean devueltos a la comunidad de la discapacidad en un formato accesible.

\footnotetext{
${ }^{5}$ Disability Human Rights Research Network (DHRRN). Protocol for Rights-based Disability Research in all Fields. February 2018

${ }^{6}$ Working paper: Guidelines for producing accessible research outputs. Prepared by Alex Holland, Yvette Maker and Anna Arstein-Kerslake. Disability Research Initiative, University of Melbourne
} 
La iniciativa puede darse a través de políticas, documentos de organizaciones de personas con discapacidad (DPOs- disabled people's organisation), a partir del diálogo formal entre investigadores y las organizaciones de personas con discapacidad, los resultados de otras investigaciones basadas en derechos humanos de cualquier lado del mundo o conclusiones del Comité sobre los Derechos de las Personas con Discapacidad.

La ejecución de la investigación se concibe bajo el principio de cesión de poder y control a la comunidad de la discapacidad. La participación se articula a través de formas e intensidades similares a las descritas en las secciones anteriores, como por ejemplo a través de grupos consultivos o colaboraciones a través de 'fellowships'. Se busca que ambas partes tengan un reconocimiento igual como contribuidores, además de una contribución económica independiente de la cobertura de gastos ocasionados por la participación en la investigación.

La respuesta a una preocupación jurídica exige que se parta de un modelo de discapacidad basado en derechos humanos (Degener, 2014), que trate de una cuestión relacionada con el ejercicio y disfrute de los derechos reconocidos en la Convención sobre los Derechos de las Personas con Discapacidad, que responda directamente a vulneraciones (real o potencial) actuales o pasadas de alguno de los derechos reconocidos en la Convención sobre los Derechos de las Personas con Discapacidad e inste al cambio social para poner fin a dicho riesgo o vulneración y que empodere a personas con discapacidad y a sus organizaciones para liderar ese disfrute de los derechos de las personas con discapacidad. Al tratarse de una iniciativa formalizada relativamente reciente, no existen muchos ejemplos. Se destaca uno relativo a la capacidad de ser juzgado como imputado (fitness to plead) en relación con el artículo 12 y 13 de la Convención sobre los Derechos de las Personas con Discapacidad (Arstein-Kerslake et al., 2019).

\section{BARRERAS, DIFICULTADES Y CRÍTICAS}

Las metodologías participativas no son de fácil implementación ni están exentas de críticas. Prácticamente todos los autores reconocen que el desarrollo de las investigaciones participativas no es linear, está lleno de complicaciones e imprevistos (Cornwall y Jenkes, 1995) o abre espacios desordenados con los que lidiar sin preaviso (Seale et al., 2015). Además, reconocen que el poder y control es raramente totalmente transferido a la comunidad o a las personas con discapacidad, sea por las obligaciones formales impuestas por las instituciones académicas, por la financiación o por las dificultades que encuentran los investigadores. Por otra parte, los investigadores pueden mostrarse reticentes a renunciar al 
control sobre la investigación, convencidos de la 'neutralidad' de su formación y superioridad del conocimiento técnico (Cornwall y Jenkes, 1995). Para los participantes, puede ser difícil contradecir o proponer alguna alternativa frente a los expertos.

La participación es costosa, requiere tiempo y no todo el mundo está interesado (Cornwall y Jenkes, 1995). Vic Finkelstein (1980) recuerda, además, que la experiencia del individuo con discapacidad no tiene por qué representar al colectivo siempre, por lo que es recomendable buscar herramientas metodológicas como la triangulación, la transparencia y permitir la participación de otras voces. Además, hay que tener en cuenta que hay personas con mayores necesidades de apoyo que no están en los grupos de autogestores o quizás nunca son invitados a participar en investigaciones (Nind, 2014; Beresford et al., 2004), por lo que es necesario reconocer esa limitación y seguir buscando la manera de oír y representar sus voces.

Otro de los retos reconocidos en la literatura es la necesidad de contar con más tiempo para realizar investigaciones participativas (Durell, 2016; Nind, 2014; Pallisera Díaz et al., 2015). Se requiere más tiempo de preparación y adaptación de los materiales y más tiempo de reacción para las personas (por ejemplo para leer y comprender la información) y para establecer una relación de confianza. En cualquiera de las modalidades de las metodologías participativas es imprescindible que los investigadores profesionales apliquen una metodología reflexiva, examinando cómo van a establecer la relación con los participantes o co-investigadores y cómo garantizar la participación y posibilidad de ser contradichos.

En caso de las personas con discapacidad intelectual, puede ser necesario ofrecer opciones concretas en lugar de preguntas abiertas. Para algunos es una limitación, mientras otros autores lo consideran un detalle menor, que no perjudica sino más bien favorece la participación de las personas con discapacidad. Conviene en este punto recordar que el objetivo no es reproducir la forma de investigación con investigadores autónomos e independientes, sino habilitar la participación de personas posiblemente sin conocimientos expertos ni experiencias previas.

El acceso a las personas con discapacidad puede ser difícil para investigadores que no tengan apenas relación con las personas con discapacidad y sus entidades sociales. Las entidades sociales pueden constituirse como filtros (gatekeepers) en la experiencia de algunos investigadores, sin embargo, otros han tenido experiencias positivas (Nind, 2014). A medida que aumente el diálogo entre las entidades y las instituciones académicas, esta 
dificultad se irá reduciendo, siempre y cuando los investigadores actúen de manera ética y no dejen un impacto negativo sobre los participantes.

Las propias instituciones a través de sus comités éticos o científicos pueden imponer trabas a la participación de personas con discapacidad intelectual, por considerarles 'vulnerables' o incapaces de dar consentimiento. Sin embargo, hay autores (Tuffrey-Wijne et al., 2008; Mietola et al., 2017) que sostienen que no es ético excluir a las personas con discapacidad intelectual de las investigaciones que puedan aportar revelaciones desde su conocimiento subjetivo sobre su realidad y cómo mejor su bienestar. Mietola et al. (2017) proponen un modelo para realizar investigación junto con personas con discapacidad intelectual sin por ello descuidar la ética y responsabilidad del investigador. La red DHRRN también ha establecido unos criterios guía para informar las cuestiones éticas, que incluye la provisión de las políticas de los comités éticos en formatos accesibles y la incorporación de los principios de la Convención sobre los Derechos de las Personas con Discapacidad a los mismos.

Se ha criticado la falta de calidad, rigor metodológico y coherencia interna de las metodologías participativas (Danieli y Woodhams, 2005). Si bien es cierto que las metodologías participativas son relativamente recientes y la disciplina de disability studies todavía es joven, los autores reconocen la complejidad de este tipo de metodologías y se han enumerado ejemplos de investigaciones rigurosas (Pallisera Díaz et al., 2015), en las que se reconocen las limitaciones y los compromisos adoptados.

Otra crítica metodológica se centra en la falta de transparencia sobre la participación efectiva y autoría en artículos científicos sujetos a revisión de pares, en los que en ocasiones no se explica en detalle cómo y en qué partes de la redacción del texto han efectivamente intervenido los investigadores con discapacidad intelectual (Strnadová y Walmsley, 2018). Estos autores señalan que dicha falta de transparencia puede plantear cuestiones de validez y apuntar a un uso simbólico de las personas con discapacidad. La complejidad de las metodologías participativas, en combinación con la dificultad de establecer voces colectivas y representativas, además de tratar de estudiar y resolver problemas de justicia social, hacen la investigación en esta área especialmente compleja. Por ello, es especialmente importante tener una planificación ética adecuada al tratarse de vidas de personas en riesgo de ser vulnerables, una conciencia de diálogo constante con las personas con discapacidad y la comunidad académica y una actitud reflexiva. 
Danieli y Woodhams (2005) critican también la imposición del modelo social, la exigencia de una metodología emancipadora o de una epistemología constructivista, sobretodo por el uso del modelo social como marco teórico. No obstante, la elección de una u otra epistemología queda a criterio del investigador y los métodos cuantitativos o cualitativos que emplee. La mayor parte de las propuestas revisadas adopta el compromiso con las personas con discapacidad tratando de hacer accesible su investigación y con intención de involucrar en el diálogo.

Los autores reconocen múltiples formas de involucrar a las personas con discapacidad y reconocen que no toda investigación se presta a un formato participativo. Las metodologías de acción participativa o investigación participativa basada en la comunidad suelen ser fundamentalmente pragmáticas, aunque deben ser sensibles (y reflexivas) a las construcciones de barreras a comunidades históricamente discriminadas, para no repetir patrones opresivos. Los principios que orientan estas metodologías tratan de atajar este riesgo, por ejemplo, mediante la permanente consulta y diálogo. En el caso de las personas con discapacidad intelectual, se ha señalado por diversos autores la falta de literatura sobre estrategias y metodologías experiencias para ofrecer apoyo a los investigadores (Pallisera Díaz et al., 2015; Bigby y Frawley, 2010).

Por otra parte, el modelo social ha permeado gran parte de las definiciones de discapacidad manejadas por la legislación de los países que han ratificado la Convención sobre los Derechos de las Personas con Discapacidad y hay autores que incluso interpretan un derecho a la participación en investigación (Ollerton y Horsfall, 2013). Hay, por tanto, una obligación de accesibilidad que traspasa la elección metodológica a una obligación legal.

Los debates reflejan, en ocasiones, aspectos capacitistas de la construcción de investigación y autoría, por ejemplo, a través de la exigencia de que cada participante aporte en todas las fases o tenga que comprender y manejar las metodologías (Mercer, 2002). Cuando se establece una propuesta participativa, los investigadores profesionales deben tener claro cuál es su rollo y qué conocimiento técnico pueden aportar. Estas críticas olvidan que, en muchas ocasiones, las personas con discapacidad no tienen oportunidad para acceder a espacios de formación y conocimiento como lo hacen las personas sin discapacidad. 
Un punto en el que coinciden todas las metodologías participativas relativas a la inclusión de personas con discapacidad es en la necesidad de ubicar el poder y el control en las personas con discapacidad (Strnadová et al., 2014).

Cornwall y Jenkes argumentan que la clave en estas metodologías (no únicamente en el área de discapacidad) está en la actitud de los investigadores y en la localización del poder. De esto depende, en buena medida, que la participación sea real y efectiva, y no se convierta en un concepto vacío o una participación simbólica. El esfuerzo personal y profesional es grande, dado que hay que manejar incertidumbre y flexibilidad. En varias etapas, la figura del investigador también se convierte en facilitador. Ambas partes enseñan y aprenden, y deben acompañarse en el proceso de ganar confianza en su capacidad de aportar conocimiento a la investigación (Cornwall y Jenkes, 1995), pues, con frecuencia, los participantes pertenecen a colectivos que tradicionalmente no son oídos ni considerados expertos (población rural o indígena, infancia, personas con discapacidad). Investigaciones realizadas por personas con discapacidad psicosocial (survivor research o mad studies) bajo un paradigma epistemológico interpretativo reconocido, encuentran muchas dificultades para ser reconocido como conocimiento válido (Sweeney y Beresford, 2019).

El valor de la investigación participativa radica en el proceso más que en el resultado (Cornwall y Jenkes, 1995), a pesar de que el objetivo de la participación es el cambio social (Strnadová et al., 2014).

La elección de metodologías participativas tiene, sin lugar a dudas, un fuerte componente politico y personal (Cornwall y Jenkes, 1995), y, aunque se puedan desarrollar investigaciones sobre las deficiencias, éstas, siguiendo la lógica establecida por el DHRRN, siempre deben estar enmarcadas dentro del avance del disfrute de los derechos de las personas con discapacidad, como puede ser el derecho a la salud, por ejemplo. Las exigencias de rigor metodológico y garantizar la participación y accesibilidad del proyecto también constituye un reto considerable (Strnadová et al., 2014).

Para hacer frente a estos retos, se han explorado otro tipo de metodologías como fotovoz (Ollerton y Horsfall, 2013; Pallisera Díaz et al., 2015), con éxito en la promoción del cambio social.

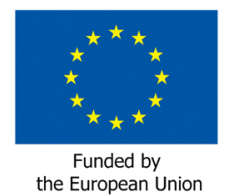

This project has received funding from the European Union's Horizon 2020 research and innovation programme under the Marie Skłodowska-Curie grant agreement No 814249;

The content of this publication represents the views of the author only and is his/her sole responsibility.

The European Commission does not accept any responsibility for use that may be made of the information it contains. 


\section{CONCLUSIÓN}

Esta revisión teórica de literatura ha recogido distintos enfoques de metodologías participativas con especial foco en las experiencias y las implicaciones para personas con discapacidad intelectual. A pesar de las dificultades reconocidas, la participación de las personas con discapacidad intelectual aporta nuevos conocimientos y ayuda a poner en evidencia barreras y actitudes capacitistas dentro de las instituciones académicas. El reto reside, en muchas ocasiones, en entender que, por una parte, la motivación de los participantes no tiene por qué coincidir con la del investigador, y que desarrollarán funciones distintas dentro de la propia investigación.

El investigador debe ser consciente del modelo de discapacidad del que parte y ser explícito de las asunciones que hace, tanto con la comunidad académica como con sus coinvestigadores.

Cada parte aporta un conocimiento distinto que enriquece el conocimiento del otro. La clave está, además, en compartir el conocimiento, alimentar la conversación y facilitar la toma de decisiones sobre el proceso de investigación y el uso de sus resultados en beneficio de las personas con discapacidad intelectual.

Las fuertes críticas a la participación más baja en decisiones entorno a metodologías puede deberse a justamente a esta división de tareas entre los investigadores y los participantes, que también ocurre, por otra parte, cuando distintos investigadores asumen tareas diferentes, o aceptamos la interpretación de datos por parte de otro investigador.

La mayoría de los artículos revisados concluyen con una visión positiva e identifican un cambio a lo largo del proceso de investigación y textos como el de Wolff (2011) sobre políticas públicas, animan a escuchar más a las personas en primera persona y ajustar así las políticas a sus realidades, necesidades y preferencias.

Así, el papel del investigador muda y el reto está en saber articular y asumir las nuevas tareas, manteniendo los principios de rigor metodológico que permita a otros entender cómo se ha llegado a determinadas conclusiones y que, en definitiva, constituye una exigencia científica, sin perder la ética de investigación y el respeto a los derechos humanos de las personas con discapacidad. 


\section{REFERENCIAS}

Arstein-Kerslake, A., Gooding, P., Mercer, S., Raymond, M. \& McSherry, B. (2019). Implementing a Participatory Human Rights-Based Research Methodology: The Unfitness to Plead Project. Journal of Human Rights Practice, 11(3), 589. doi: 10.1093/jhuman/huz034.

Beresford, B., Tozer, R., Rabiee, P., Sloper, P. (2004) .Developing an approach to involving children with autistic spectrum disorder in a social care research project. British Journal of Learning Disabilities, 32,180-85.

Biggs, S. (1989). Resource-poor farmer participation in research: a synthesis of experiences from nine national agricultural research systems. OFCOR Comparative Study Paper 3. International Service for National Agricultural Research. The Hague.

Bigby, C. y Frawley, C. (2010). Reflections on doing inclusive research in the "Making Life Good in the Community" study. Journal of Intellectual Developmental Disability, 35(2), 53-61.

Cornwall, A., Jenkes, R. (1995). What is participatory research? Social Science \& Medicine, 41(12), 1667-1676.

Danieli, A. y Woodhams, C. (2005). Emancipatory Research Methodology and Disability: A Critique. International Journal of Social Research Methodology, 8(4), 281-296, DOI: 10.1080/1364557042000232853.

Degener, T. (2014). A human rights model of disability. Routledge Handbook of Disability Law and Human Rights.

Durell, S. (2016). "Welcome to the Real World" Inclusive Research with People with Learning Disabilities: A Doctoral Journey. The Qualitative Report 21(12), 2308-2330.

Fals Borda, O. (1998). Future Action: Reflections on the Participatory Research World Congress. International Sociology 13(3), 399-402.

Finkelstein, V. (1980). Attitudes and Disabled People. New York: World Rehabilitation Fund.

Freire, P. (1973). Education for critical consciousness.

Gustavsson, A., Sandvin, J., Traustadóttir, R., Tossebro, J. (2005). Resistance, Reflection and Cnange: Nordic disability Research. Lund, Sweden: Studentlitteratur.

Hohmann, A., Shear, K. (2002). Community-Based Intervention Research: Coping With the "Noise" of Real Life in Study Design. The American Journal of Psychiatry 159, 201-7.

Israel, B., Schulz, A., Parker, E., Becker,A. (1998). "review of community-based research: assessing Partnership approaches to improve Public health." Annual Review of Public Health 19. 173-202.

Johnson, K., Traustadóttir, R. (2005). Deinstitutionalization and People with Intellectual Disabilities: In and Out of Institutions. Jessica Kingsley Publishers.

Mercer, G. (2002). ‘Emancipatory Disability Research' in Barnes, C., Oliver, M. and Barton L. (eds) "Disability Studies Today". Polity.

Mietola,R., Miettinen, S., Vehmas, S. (2017). Voiceless subjects? Research ethics and persons with profound intellectual disabilities. International Journal of Social Research Methodology, 20(3), 263-274.

Milner, P., Kelly, B. (2009). Community participation and inclusion: people with disabilities defining their place. Disability \& Society, 24(1), 47-62.

Minkler, M., Wallerstein, N. eds. (2008). Community-Based Participatory Research for Health: From Process to Outcomes (2nd edition). San francisco, CA: Jossey-bass.

Morris, J.(1992). Pride against Prejudice. London: BPCC Hazell Books.

Oliver, M. (1992). Changing the social relations of research production. Disability, Handicap \& 
Society, 7(2), 101-14

Ollerton, J., Horsfall, D. (2013). Rights to research: utilising the Convention on the Rights of Persons with Disabilities as an inclusive participatory action research tool. Disability \& Society 28(5), 616-630, DOI: 10.1080/09687599.2012.717881.

Pallisera Díaz, M., Puyaltó Rovira, C., Fullana Noell, J., Vilà Suñé M., Martin Pazos, R. (2015). Una experiencia de investigación inclusiva. Personas con discapacidad intelectual como asesoras en una investigación sobre transición a la edad adulta. Revista Iberoamericana de Educación 69, 2.

Seale, J., Nind, M., Tilley, L., Chapman, R. (2015) Negotiating a third space for participatory research with people with learning disabilities: an examination of boundaries and spatial practices. Innovation: The European Journal of Social Science Research, 28(4), 483-497, http://dx.doi.org/10.1080/13511610.2015.1081558

Stone, E., Priestley, M. (1996). Parasites, pawns and partners: disability research and the role of non-disabled researchers. British Journal of Sociology 47 (4), 699-716.

Strnadová, I., Walmsley, J., Johnson, K., Cumming, T.M. (2014). Diverse faces of inclusive research: reflecting on three research studies. Scandinavian Journal of Disability Research, DOI: 10.1080/15017419.2014.964766.

Strnadová, I., \& Walmsley, J. (2018). Peer-reviewed articles on inclusive research: Do co-researchers with intellectual disabilities have a voice? Journal of Applied Research in Intellectual Disabilities, 31(1). https://doi.org/10.1111/jar.12378

Sweeney, A., Beresford, P. (2019). Who gets to study whom: survivor research and peer review processes. Disability \& Society DOI: 10.1080/09687599.2019.1664062.

Tuffrey-Wijne, I., Bernal, J., Hollins, S. (2008). Doing research on people with learning disabilities, cancer \& dying: Ethics, possibilities and pitfalls. British Journal of Learning Disabilities, 36, 185-190.

Walmsley, J. (2001). Normalisation, Emancipatory Research and Inclusive Research in Learning Disability. Disability \& Society 16 (2): 187-205.

Ward, L., Simons, K. (1998). Practising Partnership: Involving People with Learning Difficulties in Research. British Journal of Learning Disabilities 26, 128-131.

Wolff, J. (2011). Ethics and Public Policy: A Philosophical Inquiry. London: Routledge.

Zarb, G., (1992). On the road to Damascus: First steps towards changing the relations of disability research production. Disability, Handicap and Society 7 (2), 125-138. 